\section{MS35-P3 What happens when thermal motion is frozen? A case study of polymorph stabilities for Gallic Acid Monohydrate}

Ioana Sovago ${ }^{1}$, Anna Hoser ${ }^{1}$, Arianna Lanza ${ }^{2}$, Anders Madsen ${ }^{1}$

1. University of Copenhagen, Chemistry Department

2. University of Bern, Department of Chemistry and Biochemistry

email: ioana.sovago@chem.ku.dk

There is a paramount effort from both theoretical and experimental sides in establishing the structures and properties of all the polymorphs that a system can adopt. However, many theoretical studies, including crystal structure prediction (CSP) methods are typically performed at $0 \mathrm{~K}$ and effects of thermal motion are neglected. Because vibrational effects play an important role in assessing the stability of structure, the theoretically predicted structures are often not observed experimentally. A polymorph of gallic acid monohydrate (GAM) was a target of the fifth crystal structure prediction blind test. ${ }^{\top}$ Of the six targeted systems, GAM was the only one which could not be predicted in the lowest-energy range by any of the 14 contributing groups. Here we show that GAM is a very complex system, and the lack of predictability is due to the neglect of vibrational estimates, combined with the comparison with room-temperature structures. We demonstrate that target polymorph IV is commensurately modulated at $10 \mathrm{~K}$ and disordered at higher temperatures. The GAM system is enantiotropic, leading to changes in stability as a function of temperature. Single crystal high resolution X-ray data were collected at four different temperatures $(10 \mathrm{~K}, 95 \mathrm{~K}$, $123 \mathrm{~K}$ and $175 \mathrm{~K})$. Accurate atomic and anisotropic displacement parameters were derived from aspherical atom refinement. The experimental data were further analysed by refinement of a lattice-dynamical model derived from periodic DFT/B3LYP calculations. ${ }^{2}$ This combination of theoretical modelling and experimental data allows us to derive vibrational entropies, and to assess the free energies as a function of temperature. Furthermore, some of the $\mathrm{H}$ atoms were disordered, which explains why the predicted hydrogen-bond network was ambiguous. This disorder further helps stabilizing the structure by enhancing the entropy of the system. Therefore, comparing CSP determined at $0 \mathrm{~K}$ with experimentally structures based on room temperature measurement can often be misleading and structures determined at lowest available temperature is vital for such studies.

References:

1. D. A. Bardwell, C. S. Adjiman, Y. A. Arnautova, E. Bartashevich, S. X. M. Boerrigter, D. E. Braun, A. J. Cruz-Cabeza, G. M. Day, R. G. Della Valle, G. R. Desiraju, et al., Acta Cryst. B2011, 67, 535-551.

2. A. A. Hoser, A. Ø. Madsen, ActaCrystA, 2016, A72, 206-214
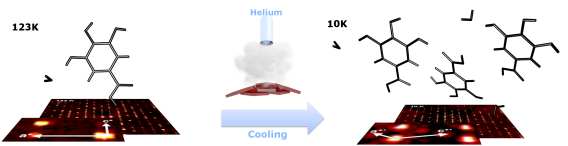

Figure 1. Gallic acid monohydrate form IV transformation under cooling. Three molecules are present in the assymetric unit at $10 \mathrm{~K}$

Keywords: lattice-dynamic, vaibrational entropy 